\title{
Internacionalización Sur-Sur: desafios y potencialidades de la Red Universitaria de la Carretera Bioceánica
}

\author{
Internacionalização Sul-Sul: desafios e potencialidades da Rede universitária do \\ Corredor Bioceânico
South South internalization: challenges and potentialities of the Biocean Route University Network \\ Ruberval Franco Maciel ${ }^{1}$ \\ Bettina Siufi ${ }^{2}$ \\ Felipe Tabilo ${ }^{3}$ \\ Mario Leiva ${ }^{4}$
}

Recebido em 10/11/2018; revisado e aprovado em 18/01/2019; aceito em 12/06/2019

DOI: http://dx.doi.org/10.20435/inter.v20iespecial.2639

\begin{abstract}
Resumen: Este artículo busca ubicar y discutir el proceso de colaboración entre las universidades brasileñas, paraguayas, argentinas y chilenas como una estrategia de internacionalización Sur-Sur, relacionada con el proyecto transnacional que involucra a los cuatro países. Es la articulación internacional y local de los asesores de relaciones internacionales de las universidades de estos países, en la constitución de la Red Universitaria de la Ruta de la Integración Latinoamericana (Unirila). La discusión se centra en situar al lector en el proceso de internacionalización e integración regional, la constitución de la red universitaria internacional, así como las potencialidades, los desafíos de integración con otros sectores involucrados y las perspectivas futuras.

Palabras clave: Carretera Bioceánica; Unirila; integración; investigación; internacionalización.

Resumo: O presente artigo buscar situar e discutir o processo de colaboração entre universidades brasileiras, paraguaias, argentinas e chilenas como uma estratégia de internacionalização sul-sul relacionada ao projeto transnacional envolvendo os quatro países. Trata-se da articulação internacional e local dos assessores de relações internacionais das universidades dos referidos países na constituição da Rede Universitária da Rota de Integração Latino Americana (Unirila). A discussão se pautará em situar o leitor sobre o processo de internacionalização e integração regional, a constituição da rede universitária internacional, bem como as potencialidades, desafios de integração com outros setores envolvidos e prospecções futuras.
\end{abstract}

Palavras-chave: Corredor Bioceânico; Unirila; integração; pesquisa; internacionalização.

Abstract: This paper aims at situating and discussing the process of collaboration among Brazilian, Paraguayan, Argentine and Chilean universities as a strategy of South-South internationalization related to a transnational project involving the four countries. It is also a result of international and local articulation of the international relations affairs offices of the universities of these countries resulting on the so called University Network of the Latin American Integration Route (Unirila). Discussion will focus on situating the reader on the process of internationalization and regional integration, on the constitution of the international university network, as well as on the potentialities, integration challenges with other sectors involved and further directions.

Keywords: Bioceanic highroad crossing; Unirila; integration; research; internationalization.

\section{INTRODUCCIÓN}

La internacionalización se ha convertido en un aspecto importante en las políticas de enseñanza superior en todo el mundo (ROCHA; MACIEL, 2018). Los significados asociados a la internacionalización son genéricos y pueden tener diferentes connotaciones de país a

\footnotetext{
${ }^{1}$ Universidade Estadual de Mato Grosso do Sul (UEMS), Campo Grande, Mato Grosso do Sul, Brasil.

${ }^{2}$ Universidade Nacional de Jujuy (UNJu), San Salvador de Jujuy, Jujuy, Argentina.

${ }^{3}$ Universidad Católica del Norte (UCN), Antofagasta, Chile.

${ }^{4}$ Universidade Nacional de Assunção (UNA), San Lorenzo, Assunção, Paraguai.
} 
país (KNIGHT, 2014 ; MACIEL; VERGARA, 2019). En este caso, Las universidades latinas son heterogéneas con distintos estados de desarrollo, tanto en los procesos de internacionalización como en su institucionalidad. Por lo que tratar de entregar una mirada uniforme al escenario de la internacionalización y los procesos de integración suele ser difícil de establecer.

En este sentido es necesario, interactuar y conocer mejor el proceso histórico de la integración latinoamericana, por medio de estudios e investigaciones de carácter regional con base en las universidades del continente. Estas redes pueden desarrollarse sin caer en dogmas ideológicos o metodológicos, propiciando el pluralismo epistemológico planteándose objetivos regionales comunes (SILVA, 2017, p. 11). Hacer una mirada crítica, pero a la vez resolutiva frente al fenómeno de la integración, teniendo como base un plan que identifique los socios estratégicos que contribuyan a la consolidación de los procesos de internacionalización e integración regional.

En la búsqueda de una propuesta legítima desde las IES, este artículo tiene por objetivo discutir la formación de una red universitaria que involucra a cuatro países latinoamericanos: Argentina, Brasil, Chile y Paraguay. Tal iniciativa resulta de la interlocución de los ministerios de relaciones exteriores de los cuatro países y que constituyen tres grupos de trabajo -gobierno, sector privado y universidades- relacionados con los trabajos que dieron soporte a la implementación del corredor bioceánico que unirá la capital Campo Grande y en la frontera el municipio de Porto Murtinho en Brasil a los puertos del norte de Chile, más específicamente las ciudades de Antofagasta e Iquique. El proyecto, en su implementación, podrá inicialmente representar dos grandes oportunidades para los investigadores de la red universitaria. Primeramente, la interlocución con los sectores privados y gubernamentales en la promoción de investigaciones que analicen los impuestos en los contextos transnacionales y, en segundo lugar, la promoción de la integración entre los investigadores de cada territorio para el enfoque en investigaciones colaborativas. En este sentido, la internacionalización es un proceso vinculado a la estrategia que responde a los objetivos de la red, cuya implementación es ordenada, sistémica y transversal. Entiendiéndola como una herramienta para fortalecer y mejorar las competencias de UNIRILA que permita lograr un posicionamiento en la región e internacional. En consecuencia, UNIRILA, se plantea como una alternativa para complementar el proceso de integración regional, pensado desde la cooperación sur-sur, y considerando la búsqueda de socios estratégicos tanto internos como externos que contribuyan a fortalecer el proceso y la consolidación de la red.

\section{2 ¿QUÉ PASA CON LAS UNIVERSIDADES EN PROCESO DE INTEGRACIÓN?}

La integración regional es un debate dinámico sujeto a constantes cambios debido a la heterogeneidad de sus actores y contextos de los países. Junto con ello, el desarrollo de políticas conjuntas de cooperación a través de compromisos vinculantes que permitan una operativa constante en pos a objetivos comunes. De todas formas, es un fenómeno que ha estado presente a lo largo de los años en la región con variados intentos por consolidar un proceso que aún está lejos de madurar. Bajo este contexto, en la región estudiada existen procesos vigentes de integración a través de las Instituciones de Educación Superior previos a la zona del Corredor Bioceánico. A continuación, se detallará brevemente las instancias por los cuales ha pasado esta región en aras a integrarse desde las universidades: Criscos (Consejo de Rectores por la Integración Centro Oeste Sudamericana) y Zicosur Universitaria (Zona de Integración del Centro Oeste de América del Sur).

La Integración en la zona que compete al Corredor Bioceánico tiene sus raíces en el intento de organizar un esquema de integración a través del Grupo Empresario Interregional del Centro 
Oeste Sudamericano (Geicos) en 1976. En una de las actividades de Geicos, en 1991 se convocó a una Asamblea Internacional celebrada en Iquique, Chile. En esa oportunidad, la Comisión 4 "Universidad, ciencia y cultura", propuso trabajar la temática de la "Educación para la integración" y crear un banco de datos para la Región, que permitiera avanzar en forma conjunta en la solución de los problemas que enfrentaban las comunidades de la Sub-región. La Universidad Arturo Prat, de Iquique, encabezó un movimiento intelectual importante a favor "de la integración regional, la dimensión cultural y educativa del desarrollo y la integración como estrategia para alcanzar el mismo, donde el conocimiento fuera una nueva e importante forma de propiedad, actuando de modo tal que los sectores sociales, habitualmente descuidados, tales como las mujeres, los jóvenes y los campesinos - entre otros - encontrarán su protagonismo en el esfuerzo integrador" (SIUFIPALOU, 1993 ). Se habló de la necesidad de elaborar un diagnóstico de situación de los sistemas educativos de la Región, para revertir las consecuencias de las asimetrías que se daban entre los Países y promover la formalización para la espontánea movilidad que se venía concretando.

A nivel superior no se daban demasiadas diferencias con los avances y retrocesos en los vínculos que se presentaban en los demás aspectos. Con mucha presencia en los discursos, pero variada resonancia en la práctica, las universidades de la Región abrieron sus puertas. Se replicaron algunas experiencias como la reunión de lquique, y en Jujuy, por ejemplo, en 1992 se realiza el Encuentro Regional del Noroeste Argentino sobre Integración Latinoamericana como un espacio de reflexión sobre las políticas generales de integración y las condiciones de inserción de las universidades a esos procesos. Se definió al hombre como eje y destinatario de políticas prospectivas de desarrollo, con clara conciencia ecológica, tendiente a resguardar el equilibrio de los sistemas naturales y definiendo que la asistencia científica, tecnológica y social que le compete en la elaboración de programas de desarrollo sostenido, debe atender particularmente a los sectores tradicionalmente más postergados.

La Comisión "Universidad, ciencia y cultura" creada por Resolución n. 647/92 de Rectorado de la Universidad Nacional de Jujuy, se integró con docentes e investigadores de todas sus Facultades bajo la coordinación de la Secretaría de Ciencia y Técnica y Estudios Regionales (Secter), con la responsabilidad de analizar profundamente el rol de las universidades en los procesos de integración latinoamericana, señalando temáticas de interés regional que quedaron establecidas como de carácter prioritario (Documento Secter-Unju sobre "Lineamientos de política científica y tecnología de la Unju). Esa Comisión, en conjunto con la Universidad Católica del Norte en Chile tuvo períodos de fecunda acción, participando activamente en la creación de Criscos en 1996 (Consejo de Rectores por la Integración Centro Oeste Sudamericana), red de universidades que alcanzó inicialmente a instituciones del norte de Argentina, Chile, de Perú y Bolivia, para luego irse incorporando Paraguay y más recientemente Ecuador. La Visión del Criscos ha sido definida como la integración de las Universidades de la Subregión, a través de la cooperación mutua, con el fin de asumir los desafíos de la globalización, mejorando su competitividad. Se ha propuesto profundizar el camino de la integración de los pueblos de Sudamérica, y en particular, de la Subregión Centro Oeste, con el propósito de ampliar las bases de cooperación académica, científico-tecnológica y cultural entre las Universidades ubicadas en la referida zona geográfica. Seminarios de investigación, redes temáticas y la edición 43 del Programa de Movilidad Estudiantil son testimonios del entendimiento entre las universidades que integran esta red.

Otro esfuerzo en pos de la integración regional se plasmó por Zicosur en cuyo marco se generó la Zicosur Universitaria (mayo 2005), que tiene como objetivos fortalecer dicha integración 
regional, propiciar el intercambio de experiencias en Metodología de Docencia, Investigación y Extensión, y fomentar y consolidar redes temáticas de docencia/investigación como herramienta de integración regional y cooperación académica. Las Asambleas de Rectores como la de sus gestores de relaciones internacionales, dan cuenta de ese espacio común de realizaciones universitarias y como Red, camina fuertemente en la concreción de actividades académicas conjuntas y la consolidación de una movilidad tanto de docentes - como de investigadores, personal administrativo y estudiantes que cubre todos los niveles del sistema.

Una vez más, la internacionalización y la integración regional son rasgos ineludibles en la universidad actual, y la constitución de Unirila es una manifestación explícita de la voluntad integradora del grupo de instituciones que la componen, con el compromiso de ser protagonistas del desarrollo de sus pueblos.

\section{LA CONSTITUCIÓN DE LA RED UNIVERSITARIA DEL CORREDOR BIOCEÁNICO}

Para entender la constitución de la red universitaria Unirila del Corredor Bioceánico, es necesario tomar en consideración los procesos de integración en la región latinoamericana bajo el concepto de cooperación sur-sur como un fenómeno dinámico. En este sentido, Latinoamérica pasa por tres etapas reconocidas de regionalización para la integración de sus países: el primero es el Viejo Regionalismo, entre la década de los sesenta y mediados de los ochenta, quien basa sus políticas en la importancia de la unión aduanera para facilitar las relaciones comerciales y por otra parte, la generación de un entramado industrial que mitigara la dependencia latinoamericana con los centro económicos del mundo. El segundo, Regionalismo Abierto, durante los noventa hasta comienzos del nuevo siglo, concentrado principalmente en la apertura de mercados, a través de tratados de libre comercio, aperturas fronterizas, infraestructuras regionales y algunas cuestiones de política ambiental y laboral. Sin embargo, este modelo basado en los principios de neoliberalismo, produjo grandes diferencias sociopolíticas en la región, que se tradujeron en la crisis de la deuda. Finalmente, viene una tercera generación, el Regionalismo Post-Liberal, a principios del siglo XXI, que responde al agotamiento del modelo liberal de las anteriores e intenta buscar una reinterpretación de los procesos de integración regional (Ojeda; Surasky, 2014, p. 25).

En relación a ello, se considera: "[...] una visión crítica de las reformas liberales y de las experiencias de integración de los noventa [y] señala el exceso de énfasis conferido por esos esquemas bilaterales y subregionales a las cuestiones comerciales, lo que habría llevado a ignorar temas relacionados con las asimetrías estructurales entre los países miembros y, de manera más general, a dejar de lado preocupaciones como las dimensiones productivas y 'de desarrollo' de los procesos de integración” (OJEDA; SURASKY 2014, p. 26). Sobre este marco, se van generando nuevos sistemas de integración regional, sumando diferentes actores cuyos roles se complementan en la búsqueda de un camino común, en el concierto del nuevo regionalismo Post-Liberal. En este sentido, el concepto del Corredor Bioceánico sienta sus bases para el desarrollo de un esquema de integración más acotado, pero que busca resultados concretos de forma integral, buscando suavizar las diferencias entre los Países miembros, mitigar los efectos negativos de la globalización, la presencia de una agenda política con actores estatales, más que a la liberalización de las interacciones comerciales entre privados y una mayor preocupación por los "cuellos de botella", entendidos como: "[...] las carencias de la infraestructura regional, con el objeto de mejorar la articulación de los mercados regionales y facilitar el acceso a mercados 
externos" (OJEDA; SURASKY 2014, p. 28-30).

La Declaración Presidencial de Asunción, aprobada en diciembre de 2015, por los Altos Mandatarios de Argentina, Brasil, Chile y Paraguay, reflejó el propósito firme de los cuatro países en implantar un Corredor Vial Bioceánico, con origen en Campo Grande y Porto Murtinho (Brasil), pasando por Carmelo Peralta, Mariscal Estigarribia, Pozo Hondo (Paraguay), Misión La Paz,Tartagal, Jujuy, Salta (Argentina), Sico, Jama hasta alcanzar los puertos en el Pacífico de Antofagasta-Mejillones e Iquique (Chile). Como resultado de la declaración presidencial, fueron creadas tres instancias de trabajo - gubernamental, académica y empresarial - y se realizaron en los últimos dos años diversas reuniones presenciales y por video conferencia para dar secuencia a la agenda presidencial. El elevado apoyo político al proyecto fue renovado por la Declaración de Brasilia, aprobada en diciembre de 2017.

La red universitaria del Corredor Bioceánico, en su vertiente brasileña, fue constituida por representantes de tres universidades públicas, una institución federal y dos universidades privadas del Estado de Mato Grosso do Sul (UEMS, UFMS, IFMS, UCDB y UNIDERP). La red fue constituida en julio de 2016 en la ciudad de Campo Grande en el / Seminario del Corredor Bioceánico y sus acciones son apoyadas por el Consejo de Rectores de las Instituciones de Enseñanza Superior de Mato Grosso do Sul (CRIE-MS). Las instituciones similares de los demás países que pertenecen a la red universitaria son:

Paraguay: Universidad Nacional de Asunción;

Argentina: Universidad Nacional de Salta; Universidad Nacional de Jujuy, Universidad Católica de Salta;

Chile: Universidad Católica del Norte y Universidad de Antofagasta.

La red universitaria del Corredor Bioceánico, como instancia de trabajo, hasta la presente fecha está bajo la coordinación de la Universidad Estadual de Mato Grosso do Sul (UEMS).

El proceso de definición de acciones a realizar por la red universitaria en apoyo a la implantación del Corredor Bioceánico, según el entendimiento entre las universidades de los cuatro países (noviembre de 2017), avanzó en el I Seminario de la Red Universitaria de la Ruta de Integración Latinoamericana, realizado en la Universidad Estadual de Mato Grosso do Sul y en la Universidad Católica Dom Bosco. Allí fue creada la Unirila, en la que se priorizaron tres ejes temáticos, a saber: I) Impactos sociales; II) Desarrollo local y turismo; y III) Internacionalización y potencialidades académicas. En lo que se refiere al tercer grupo de trabajo - Internacionalización y potencialidades académicas-, conformado por representantes de las oficinas de relaciones internacionales de las referidas universidades, se consensuaron tres acciones principales: I) Elaborar la reglamentación de funcionamiento de la Unirila; II) Constituir un Comité responsable por la gestión de la Unirila, conformado por representantes de cada Universidad; y III) Mapear las potencialidades de enseñanza, investigación y extensión de las Universidades participantes de la Red.

Creemos que esa articulación dentro de la red universitaria, puede abrir espacio para una acción inédita con la creación de una red internacional de investigación, integrada por los cuatro países y dirigida a proporcionar apoyo a las acciones tanto para las cuestiones gubernamentales cuanto como las empresariales. Como prospección, sugerimos seguir una propuesta de posibilidad de articulación del grupo de trabajo de internacionalización y potencialidades académicas, con los dos otros grupos de trabajo de la Unirila. 


\section{POTENCIALIDADES Y DESAFÍOS DE UNIRILA EN LA INTEGRACIÓN REGIONAL}

Bajo el marco de la Declaración de Asunción sobre Corredores Bioceánicos del 2015, las partes reconocieron el potencial complementario que tienen sus economías, conectando a través de vías y carreteras el tránsito de personas y productos para agilizar y hacer eficiente su integración. Esto finalmente apuntará a mejorar la competitividad de los productos de la región en los mercados de Europa y sobretodo el Asia Pacífico, así como las condiciones socio-económicas de los miembros. Chile define que a través de sus puertos del norte, contribuirá como plataforma logística al comercio internacional de bienes y servicios de los miembros del Corredor. Bajo este contexto, los gobiernos instruyeron a sus Ministerios de Relaciones Exteriores a conformar grupos de trabajo entre los cuatro países con representantes de los ministerios involucrados en el proyecto, según las áreas requeridas, así como otras instituciones para iniciar estudios técnicos y recomendaciones en los ámbitos del Corredor Bioceánico (Declaración de Asunción, Mercosur, 2015). Entre el grupo resultante, se decidió incrementar el número de miembros del Grupo de Universidades, invitando a otras instituciones que comparten el espacio del Corredor.

En la III Reunión de los grupos de trabajo conformados (Jujuy/Argentina, agosto de 2016) se identificaron las necesidades para desarrollar el Corredor (Conexión Intal, BID, 2017). Finalmente, en la IV Reunión (Salta/Argentina, junio/2018) quedaron consolidadas las siguientes mesas:

a) Plan Piloto para la Simplificación de los Procedimientos en Fronteras: El trabajo se concentra en generar un acceso integrado y anticipado a la información entre los cuatro países. Diseñar una propuesta de agilización de procedimientos aduaneros, inspección y control fronterizo eficiente y eficaz. En seguridad, se pretende diseñar un procedimiento de control zoo y fitosanitario y movimiento de carga y personas único.

b) Obras Públicas, Logística y Transportes: el objetivo es diseñar un plan y cronograma de las infraestructuras que se definan necesarias para articular el corredor, teniendo como meta la conclusión de las obras al año 2021 y un grupo multilateral para dar seguimiento al avance de proyectos. Adicionalmente, se trabaja sobre la complementación de los modos de transporte y desarrollo de centros logísticos, cadenas de valor y proyectos de integración productiva.

c) Producción y Comercio: Se encarga de realizar un "Mapa de Actores Productivos para la Integración del Corredor Bioceánico" y articular acciones necesarias para dar origen a la "Red de Integración Productiva del Corredor Bioceánico" que involucre a todos los actores institucionales, públicos y privados.

d) Red Universidades: En esta oportunidad se definieron los objetivos siguientes: Proveer información sobre las problemáticas sociales, económicas, políticas, y de servicios; crear una base de datos para sistematizar dicha información; y publicar la misma en la página web del corredor (aún en desarrollo).

\section{5 ¿CUÁL ES EL ROL DE LAS UNIVERSIDADES EN ESTE PROCESO DE INTEGRACIÓN?}

Uno de los roles de las universidades en este proceso puede ser el de constituirse en articuladoras en los procesos de integración, además de un importante recurso en la formación de redes internacionales entre instituciones para la movilidad de capital humano especializado, el desarrollo de investigación conjunta en temas específicos de interés de los grupos de trabajo; y la presentación de proyectos de transferencia tecnológica y de emprendimiento, que contribuyan a las acciones de los países que hayan decidido iniciar relaciones en un espacio multilateral. 
Desde la perspectiva expuesta, el rol de las universidades en la actualidad es más que la enseñanza e investigación, pues bajo el concepto de triple hélice: Gobierno, Empresa y Universidad, las Instituciones de Educación Superior han diversificado su quehacer en el desarrollo regional (TRIPPL; SINOZIC; BIRKBECK, 2014). Los mismos Autores destacan la figura de "Universidad comprometida", que ya había sido señalada por las autoridades participantes del II Encuentro Internacional de Rectores Universia (Guadalajara / México, junio 2010)

En cuanto a la investigación aplicada, las universidades siguen determinadas prioridades regionales de desarrollo detectadas por las autoridades gubernamentales locales en coordinación con la autoridad central. En el caso particular de la Unión Europea a través de sus programas de Horizonte 2020, entregan fondos e incentivos para el desarrollo de estas actividades que no solamente están acotadas a las universidades, sino que van dirigidas tanto a las organizaciones de sociedad civil, como a las empresas locales, en razón a desarrollar las prioridades de región. Finalmente, estas actividades generan que tanto las universidades como otros actores locales, puedan aportar en la formación de políticas públicas regionales que apunten al desarrollo del territorio.

En este caso, Unirila se orienta a establecer compromisos con las regiones que la conforman, no solo para la formación de capital humano profesional acorde a las necesidades locales y nacionales, sino proyectados a desarrollar y servir de instrumento de apoyo y soluciones conjuntas a problemas de tipo económico, social y tecnológico que se puedan presentar con la implementación del proyecto de integración enmarcado por el Corredor Biocéanico. En cuanto a las potencialidades, Unirila presenta un número importante de instituciones heterogéneas que ofrecen fortalezas en sus áreas contenidas en los distintos Grupos de Trabajo, organizados por el sector público en el marco del Corredor, pudiendo generar complementariedad entre los distintos proyectos.

Por otra parte, esta instancia fortalece las zonas de integración periféricas en el proceso de regionalización, entregando mayor visibilidad a instituciones que se encuentran alejadas de los polos comerciales más relevantes de los Países participantes. En este sentido, Unirila se puede transformar a través de la investigación aplicada y la transferencia tecnológica, en un promotor de la integración regional, contribuyendo a posicionar a los países socios del Corredor, en el Asia Pacífico como macroespacio económico, social y cultural. Sin embargo, el riesgo que pudiera estar presentando Unirila, es que los objetivos de las universidades y el desarrollo regional en torno al Corredor, no se vinculen con los objetivos que buscan los gobiernos en este proceso, siendo importante crear espacios de participación donde se dialogue sobre los avances que realizan dichos Gobiernos en el desarrollo del Corredor, para que el sentido de la asociación de universidades sea coherente a las expectativas de las autoridades y de provecho la contribución de las instituciones educativas al proyecto.

Es entonces que Unirila se define como la asociación entre entidades de educación superior de gestión pública y/o privada, que comparten un objetivo regional de integración, conformada por universidades de los cuatro países que atraviesa el Corredor. De ahí que, la principal tarea de Unirila es apoyar las mesas de trabajo y realizar propuestas fundamentadas a sus demandas específicas. Considerando lo anterior, Unirila se propone organizar grupos de expertos universitarios de los países integrantes que den apoyo de los grupos ministeriales, desarrollando investigación, estudios de mercado, evaluación de impactos y medidas de mitigación, relacionado a la factibilidad económica, medioambiental, social y comercial y con transferencia tecnológica, tomando en cuenta los requerimientos del Corredor Bioceánico. 
En la actualidad y a lo largo del proceso de integración del Corredor, las universidades fundadoras han trabajado en fortalecer sus vínculos e ir incorporando aquellas otras que si bien no estuvieron desde los inicios del Corredor, han manifestado su voluntad de pertenecer a la Red. Además, se ha avanzado en la elaboración de sus propias regulaciones, consensuando los principios que posibilitan la convergencia y armonizando la diversidad propia de su condición de instituciones educativas autónomas.

Por ello, es necesario reunir los esfuerzos para facilitar la interacción entre las instituciones - a través de programas conjuntos de movilidad, tanto de docentes como de estudiantes y personal administrativo-, como también, vehiculizar proyectos plurinacionales de investigación y/o extension - que permita contribuir al logro de los objetivos de las mesas de trabajo, para hacer cumplir la función de articulador y conector de la integración de manera transversal. Finalmente, Unirila debe contribuir a que las universidades salgan: "[...] a la palestra pública y convertirse no solo en el lugar de la respuesta a los problemas regionales sino ser un actor creativo de la integración con miras a la eliminación de todas las asimetrías de nuestros pueblos" (SILVA, 2017, p. 11).

\section{CONSIDERACIONES FINALES}

Las universidades en el escenario latinoamericano han tomado distintos roles en el desarrollo de la sociedad. En algunos casos se han convertido en la punta de lanza de procesos políticosociales y en otras han sido centro de desarrollo crítico frente a los distintos acontecimientos, a lo largo de los siglos en nuestra región. Como hemos apreciado, el rol de las universidades en la integración de los países de Latinoamérica ha ocupado un espacio como centros de conocimiento y formación del capital humano avanzado que de una u otra forma contribuyen al desarrollo de sus respectivos países. Sin embargo, en los temas relacionados a la integración latinoamericana, muchas veces su presencia se ha limitado a generar relaciones entre las universidades relegadas de los procesos políticos de la integración o procesos regionales entre gobiernos y otros actores.

Bajo este marco, es que Unirila hace un llamado a trabajar en conjunto con las mesas de trabajo, en el sentido de poder contribuir al proceso de integración no solamente en un marco interuniversitario, sino también para cooperar con los procesos estratégicos de los Países miembros. La presencia de Unirila puede contribuir a las relaciones entre Gobierno, Empresa Privada y Sociedad Civil, para comprender el fenómeno de la integración y transformarse en un eje articulador para el desarrollo del Corredor Bioceánico.

Finalmente, al crecer y madurar los espacios de participación y coordinación en el proceso de integración, las universidades de Unirila podrán realizar proyectos de investigación y colaboración como productos deseables y concretos en el proceso del Corredor Bioceánico, que como parte de sus funciones tengan la generación de cadenas de valor que maximicen las potencialidades de la Región y su apertura al mundo.

\section{REFERÊNCIAS}

BANCO INTERAMERICANO DE DESARROLLO (BID). Conexión Intal. Corredor Vial entre el Atlántico y el Pacífico. 11 jul. 2017. Disponible: http://conexionintal.iadb.org/2017/07/11/corredor-vial-entre-elatlantico-y-el-pacifico/. Acceso en: 20 jun. 2019. 
CHILE. Ministerio de Relaciones Exteriores. Declaración de Asunción sobre Corredores Bioceánicos, Asunción: MINREL, 21 dic. 2015. Disponible: https://minrel.gob.cl/minrel/site/artic/20151221/ asocfile/20151221164909/declaraci_n_de_asunci_n_sobre_corredores_bioce_nicos.pdf. Acceso en: 10 jun. 2019.

KNIGHT, J. Internationalization remodeled: Definitions, rationales, and approaches. Journal of Studies in International Education, v. 8, n. 1, p. 5-31, 2014.

MACIEL, R. F.; VERGARA, V. S. Um olhar situado sobre o papel da língua no English Club e no Curso de Medicina. Revista Oregon, UFRGS, 2019.

OJEDA, T. O. M.; SURASKY, J. La Cooperación Sur-Sur y los Procesos de Regionalización e integración en América Latina a comienzos de Is siglo XXI. En: AYLLÓN, B., OJEDA, T.; SURASKY, J. (Coord.). Cooperación Sur-Sur: regionalismos e integración en América Latina. Instituto Universitario de Desarrollo y Cooperación. Madrid, España: Ed. Catarata, dic. 2014. p. 19-33. Disponible: https://www.researchgate. net/publication/283939533_Cooperacion_Sur_-_Sur_Regionalismos_e_Integracion_en_America_Latina. Acceso en: 20 jun. 2019.

REUNIÓN DE GRUPOS DE TRABAJO CORREDOR BIOCEÁNICO, 6., junio 2018., Apuntes Mesa de Trabajo universidades. Universidad Católica del Norte, 2018.

ROCHA, C. H.; MACIEL, R. F. Internacionalização do ensino superior. En: MACIEL, R. F; TILIO, R.; JESUS, D. M.; BARROS, A. L. E. C. (Org.). Linguística aplicada para além das fronteiras. Campina: Pontes, 2018.

SILVA, E. Cultura de paz, integración latinoamericana y universidades. Cultura de Paz. Managua, Nicaragua, v. 23, n. 71, enero/abr. 2017. Disponible: https://www.researchgate.net/publication/317060171_Cultura_ de_Paz_integracion_latinoamericana_y_universidades. Acceso en: 15 jun. 2019.

SIUFI, B.; PALOU, R. La educación como ámbito de encuentro regional: una realidad sostenida. Trabajo presentado en el Primer Seminario Internacional de Integración Sub-regional. Iquique (Chile), 1993.

TRIPPL, M., SINOZIC, T., BIRKBECK, H. L. S., The role of universities in regional development: conceptual models and policy institutions in the UK, Sweden and Austria. CIRCLE, Lund University, July 2014. Centre for Innovation, Research and Competence in the Learning Economy. CIRCLE. Sweden.

\section{Sobre los autores:}

Ruberval Franco Maciel - Postdoctor en Educación Bilingüe por la City University of New York, en los Estados Unidos. Doctor en Letras por la Universidade de São Paulo (USP), com pasantía doctoral por la University de Manitoba, no Canadá. Máster em Linguística Aplicada por la University of Reading, Inglaterra. Asesor en Relaciones Internacionales y Movilidad Académica de la Universidade Estadual de Mato Grosso do Sul (UEMS).E-mail: ruberval.maciel@gmail.com, Orcid: http://orcid.org/0000-0003-0373-1047

Bettina Siufi - Doctora por la Universidad de Murcia (España). Magister Scientiae en Metodología de la Investigación Científica y Técnica. Diplomada en Pensamiento Complejo. Licenciada y Profesora de Filosofía. Coordinadora de la Oficina de Programas de Internacionalización e Integración Regional Universidade Nacional de Jujuy (UNJu), Argentina. Secretaria Permanente de Consejo de Rectores por la Integracion de la Subregión Centro Oeste de Sudamérica (CRISCOS). E-mail: bsiufi@gmail.com, Orcid: http://orcid.org/0000-0001-7654-7262 
Felipe Tabilo - M. A. Ciencias Políticas Meción Relaciones Internacionales por la Pontificia Universidad Católica de Chile. Licenciado en Historia por la Universidad Adolfo Ibáñez. Coordinador Redes Internacionales, Dirección Relaciones Institucionales, Universidad Católica del Norte. Educación. Email: redesinternacionales@ucn.cl, Orcid: http://orcid.org/0000-0002-8619-8905

Mario Leiva - Doctor en Administración de Empresas por la Universidad Autónoma de Asunción (UAA). Master en Gestión de Empresas por la Universidad Autónoma de Asunción (UAA). Master en Gestión de Empresas por la Escuela de Administración de Empresas (EAE), Barcelona, España. Licenciado en Tecnología de Producción por la Universidad Nacional de Asunción (UNA). Profesor titular de la Universidad Nacional de Asunción, Facultad de Ciencias Exactas y Naturales, Departamento de Tecnología de Producción.E-mail:mleiva137@gmail.com, mleiva@facen.una.py, Orcid:http://orcid.org/0000-0003-1416-7162 